\title{
Schützt Zoledronsäure vor Kollaps des Femurkopfs?
}

\author{
Lee et al. untersuchten in einer randomisierten Multicenter- \\ Studie, ob die präventive Gabe des Bisphosphats Zoledronsäu- \\ re die Infraktion des Hüftkopfs bei Osteonekrose verzögern \\ kann. \\ Lee YK, Ha YC, Cho Y] et al. Does Zoledronate Prevent Femoral Head Collapse from Os- \\ teonecrosis? A Prospective, Randomized, Open-Label, Multicenter Study. J Bone Joint \\ Surg Am 2015; 97: 1142-1148
}

\section{Einleitung}

$\nabla$

Die Osteonekrose des Femurkopfs führt häufig zur Infraktion des nekrotischen Areals mit anschließender Degeneration des Hüftgelenkes und ist die häufigste Ursache für die Hüftendoprothetik bei jungen Erwachsenen. Es wurde wiederholt berichtet, dass die Einnahme von Bisphosphonaten diesen Prozess verzögern kann. Diese 2-jährige prospektive randomisierte open-label Multicenter-Studie, sollte klären, ob Zoledronat die Infraktion des Femurkopfs bei Osteonekrose verhindern kann und die Notwendigkeit der Hüftendoprothetik reduziert.

\section{Methodik \\ $\nabla$}

110 Patienten mit nicht traumatischer Osteonekrose des Hüftkopfes (SteinbergStadium I oder II) und einem nekrotischen Bereich von $\geq 30 \%$ wurden in diese Studie eingeschlossen und randomisiert der Zoledronsäure- $(n=55)$ bzw. der Kontrollgruppe $(n=55)$ zugeteilt. Die Patienten in der Zoledronat-Gruppe erhielten $5 \mathrm{mg} / \mathrm{Jahr}$ Zoledronat intravenös für 2 Jahre, während Patienten in der Kontrollgruppe dieses Medikament nicht erhielten. Der primäre Endpunkt war die Überlebensrate in Bezug auf das Auftreten der Infraktion des Femurkopfes ( $\geq 2 \mathrm{~mm}$ ). Die Patienten wurden für mindestens 2 Jahre nach Studieneinschluss beobachtet.

\section{Ergebnisse}

Während des 2-Jahres-Follow-up, kam es zum Kollaps bei 29 Femurköpfen in der Zoledronat-Gruppe und bei 22 in der Kontrollgruppe $(p>0,05)$. Eine Hüftendopro- these erhielten 19 Patienten in der Zoledronat-Gruppe und 20 in der Kontrollgruppe $(p>0,05)$.

Zoledronat verhindert nicht die Infraktion des Hüftkopfes bei Osteonekrose mit Steinberg-Stadium I oder II bei einem mittleren bis großen nekrotischen Bereich, und reduziert nicht Anzahl der dadurch bedingten Hüftendoprothesen.

\section{Kommentar \\ $\nabla$}

Die präventive Behandlung der Osteonekrose des Femurkopfs mit Bisphosphonaten ist nach der vorliegenden gut geplanten randomisierten Multicenter-Studie nicht indiziert. Es fanden sich keine klinischen Vorteile durch Zoledronat-Gabe im Vergleich zur Kontrolle. Auch die Anzahl der Hüftendoprothesen wurde nicht reduziert. Somit gibt es weiterhin keine vielversprechende nicht-invasive Therapiemöglichkeit der Osteonekrose des Hüftkopfes bei mittlerem bis großem Nekroseareal, um operative Verfahren (Dekompression, Spongiosatransplantation, Osteotomie) zu umgehen. Ob ein anderes Bisphosphonat bessere Ergebnisse erzielen würde, bleibt stark zu bezweifeln.

\section{Dr. med. Robert Rotter}

Abteilung für Unfall-, Hand- und Wieder-

herstellungschirurgie

Universitätsmedizin Rostock

Robert.rotter@med.uni-rostock.de 